\title{
The ethnobotany of medicinal plants in supporting the family health in Turgo, Yogyakarta, Indonesia
}

\author{
MAIZER SAID NAHDI, IKA NUGRAHENI ARI MARTIWI, DISCA CAHYARI ARSYAH \\ Biology Education Program, Department of Biology, Faculty of Science and Technology, Universitas Islam Negeri Sunan Kalijaga. Jl. Marsda \\ Adisucipto No. 1, Yogyakarta 55281, Indonesia. Tel. +62-274-540971, Fax. +62-274-519739`email: maizersn@yahoo.co.id
}

Manuscript received: 5 March 2016. Revision accepted: 27 October 2016.

\begin{abstract}
Nahdi MS, Martiwi INA, Arsyah DC. 2016. The ethnobotany of medicinal plants in supporting the family health in Turgo, Yogyakarta, Indonesia. Biodiversitas 17: 900-906. The knowledge of healing using medicinal plants among the people of Turgo Hamlet, Purwobinangun, Sleman, Yogyakarta, Indonesia has been inherited from generation to generation. This knowledge must be studied and preserved. This study was conducted from January to June 2014 with an objective of studying the ethnobotany of medicinal plants in Turgo Hamlet community, including the local knowledge of medicinal plants to support the family health, the parts of plants used as medicines and the processing of medicinal plants. Qualitative and quantitative methods were used to collect data, using in-depth interview with 40 respondents selected purposively. The results showed that the people of Turgo Hamlet used 69 plant species from 36 families as medicinal plants. The most used part of plant was leaf (51\%), followed by fruit (15\%), rhizome (11\%), stem (5\%), root (4\%), sap (3\%), flower (3\%), all parts (3\%), tubers (3\%), and endosperm (2\%). The medicinal plants were processed or directly used as medicines. Most of the medicinal plants were boiled (62\%); others were smeared on skin (15\%), directly consumed (12\%), cooked (4\%), used for bathing (3\%), burned (3\%), and crushed using a kitchen blender (1\%). The medicinal plants were used for external (33\%) and internal (67\%) diseases.
\end{abstract}

Keywords: Generation, heritage, in-depth interview, plant organs, purposive sampling

\section{INTRODUCTION}

It is important to study ethnobotany because it is related to sustainable rural development in a region. Ethnobotany can also be used to know the dynamics of traditional ecological knowledge as an effort for biodiversity conservation in the future (Pieroni et al. 2014). Conservation of specific mountain ecosystem requires a multidisciplinary approach, so that the utilization of the ecosystem can be done on a sustainable basis in order to preserve the ecosystem service as a life supporting system (Idolo et al. 2010; Kandari et al. 2012; Khan et al. 2013).

Indonesia has abundant natural resources, including many species of plants, more than 2,039 of which have medicinal effect (Zuhud 2009). Each community has their own knowledge of the use of plants, not only for economic and cultural purposes but also for medicinal use (Kandari et al. 2012; Matthew et al. 2013). The current medicines can be divided into two categories, namely modern and traditional medicines (Muhammad 2000). Modern medicines are produced by pharmaceutical industries using sterile and reactive chemicals. On the other hand, traditional medicines are usually processed using a simple technology, based on recipes inherited from generation to generation, following local traditions and belief. Some are based on magical power, while others are based on traditional knowledge. Although they have slow reaction, traditional medicines have some benefits: they are cheaper, easy to get, easy to digest and do not have side effects (Bodeker 2000; Martin 2004). The use of natural substances as medicines and other products has been increasing. The substances have been used by lower-and middle-class families especially for prevention and curation of diseases, and rehabilitation and promotion of health. The researches on medicinal plants are increasing along with the increasing awareness of people of healthy life, and the demand on food has shifted. People not only care about the taste but also the effect of food on health (Setyowati 2010).

Turgo Hamlet is located in the slope of Mount Merapi with a total area of 200 hectares. It has an ecosystem influenced by the people's wide knowledge of medicinal plants. So, many species of medicinal plants are found in the hamlet and the people use the organs of the plants or the whole plants to maintain the family health. Their livelihood as farmers is in line with their hobby to preserve medicinal plants and to consume traditional medicinal plants for curation of diseases and maintenance of family health. Currently, there is a shift of their opinion regarding medicines. They know modern medicines and some of them abandon traditional medicines and prefer to use modern medicines which can react quickly. However, some of them, especially the native residents and farmers, still care about traditional medicines and preserve traditional healing.

Based on the above background, it is necessary to develop the local wisdom of Turgo Hamlet community in using belief and knowledge of medicinal plants as traditional healing heritage to maintain family health. There are other reasons why the medicinal plants in Turgo Hamlet needs to be documented: the potential of medicinal 
plants is high; some residents still have knowledge of medicinal plants; there is land ecologically suitable for cultivation of medicinal plants which will help biodiversity conservation. Based on the above reasons, it is important to do research on the ethnobotany of medicinal plants in Turgo Purbowinangun Village, Yogyakarta, Indonesia, in order to know the local knowledge of medicinal plants to support family health, the parts of plants used as medicines and the processing of medicinal plants.

\section{MATERIALS AND METHODS}

This study was conducted in Turgo Hamlet, Purwobinangun, Pakem, Sleman, Yogyakarta, Indonesia from January to June 2014. This is the highest hamlet found in this rural area, located only $7 \mathrm{~km}$ from Mount Merapi. Geographically, it is located in $07^{\circ} 35.668 \mathrm{~S}$ and $110^{\circ} 25.118 \mathrm{E}$, with an altitude of $900-1000 \mathrm{~m}$ above sea level (Figure 1). The tools used in this study were: recorder, digital camera, note books, pens, scissors, plastic bags and questionnaire. The materials used were all medicinal plants found in the study site. The plants were identified using Steenis (1972) and Backer (1973).

A combination of qualitative and quantitative methods were used to collect information of ethnobotany of medicinal plants to support family health in order to know local knowledge of medicinal plants, the parts of plants used as medicines and the processing of medicinal plants. Ethnobotanical data were gathered through in-depth interview with respondents using open questionnaire. Respondents were selected using purposive sampling, based on certain criteria.
The number of respondents was 40. They were residents of Turgo Hamlet, consisting of native residents who were still concerned with medicinal plants, represented by 2 traditional midwife (5\%), 7 old people (17.5\%) and local community represented by 10 members of farmer group (25\%), native residents represented by 16 medicinal plant farmers (40\%). Based on their education, $40 \%$ of respondents graduated from elementary school, $30 \%$ from junior high school, and 30\% from senior high school. Based on gender, $65 \%$ of respondents were female and $35 \%$ were male. Most respondents were farmers (85\%), and the rest were civil servants and traders (15\%) (Figure 2).

Information of medicinal plants were obtained from the community. Then the plants were collected from home gardens and forest around the hamlet. Every plant was identified using local name and scientific name. Unidentified plants were photographed and made into herbarium for further identification by botanist in the Laboratory of Botany and Ecology, Faculty of Science and Technology, University Islam Negeri Sunan Kalijaga Yogyakarta, Indonesia. The results of plant inventory were analyzed descriptively and quantitatively using tables.

\section{RESULTS AND DISCUSSION}

The results showed that the people of Turgo Hamlet have used 69 plant species, from 36 families as medicines, consisting of 14 species of trees (20\%), 22 of shrubs (31\%), 4 of lianas (5\%), 26 of herbs (37\%), and 3 of grasses (4\%) (Figure 3). Family Zingiberaceae (10.14\%) was the most used plants to cure various diseases and to maintain health and skin beauty (Tables 1 and 2).
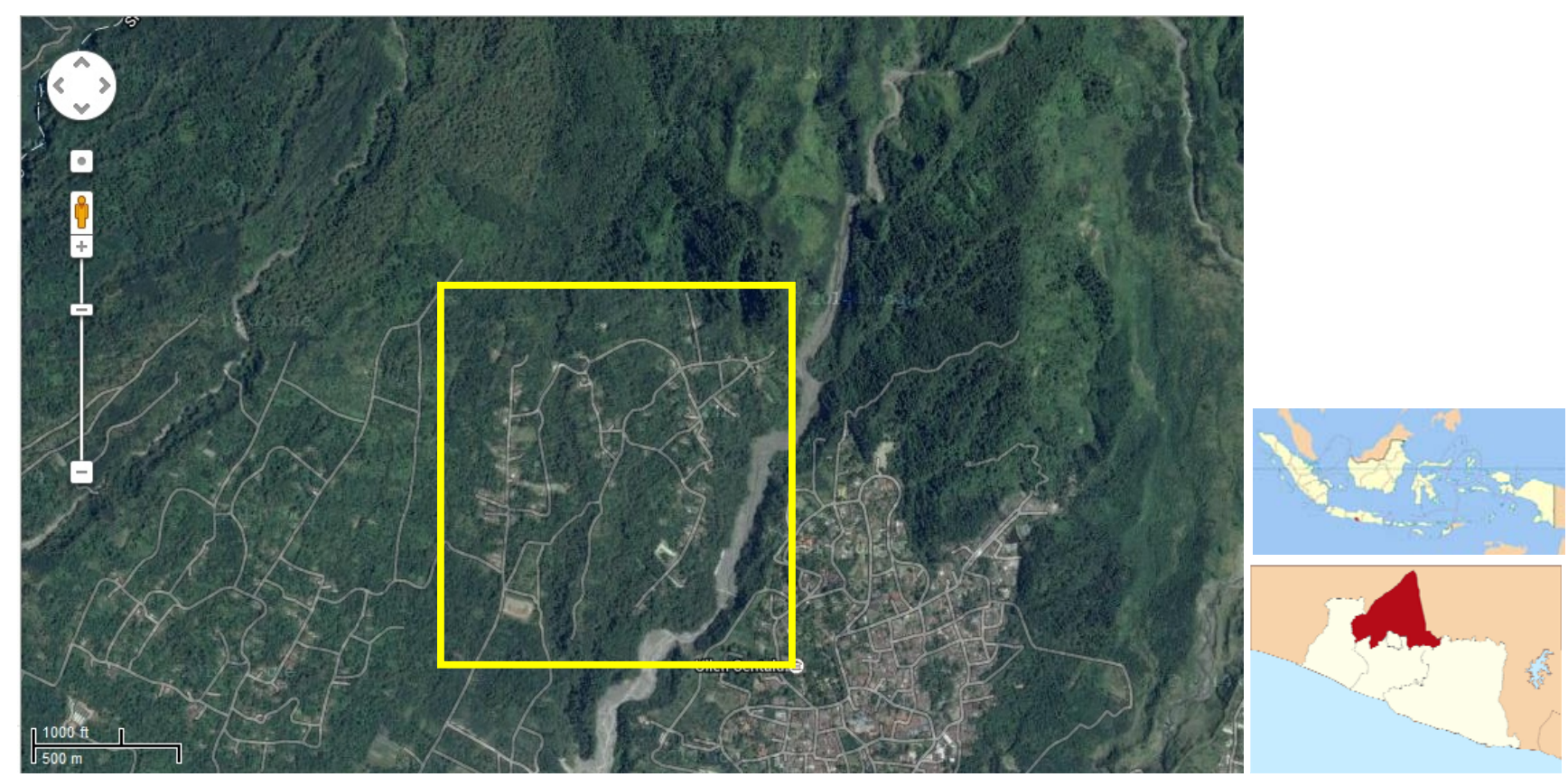

Figure 1. Location of study on the south slope of Mount Merapi, i.e.: Turgo Hamlet, Purwobinangun Village, District of Sleman, Yogyakarta, Indonesia 


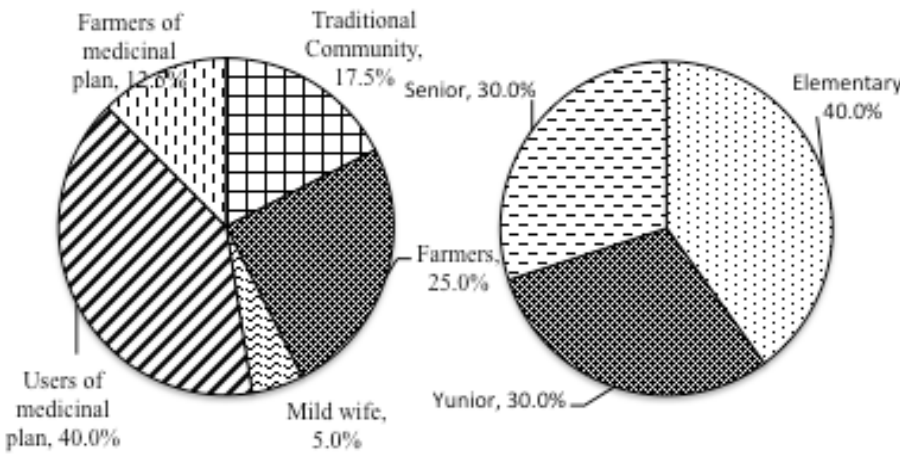

A

B

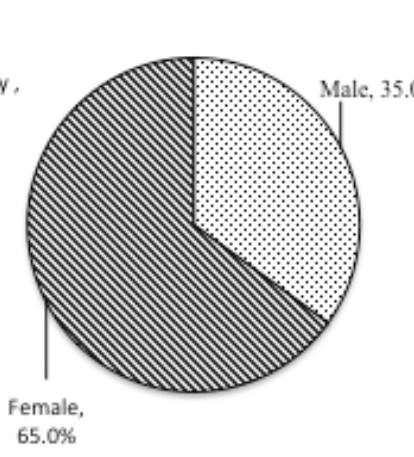

C

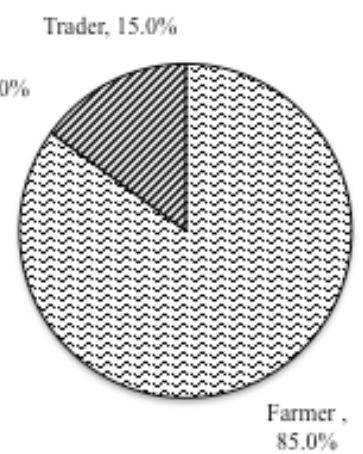

D

Figure 2. Qualification of respondents based on: A. Representation of community, consisting of native residents, traditional community and local community, B. Education level, C. Gender, and D. Occupation
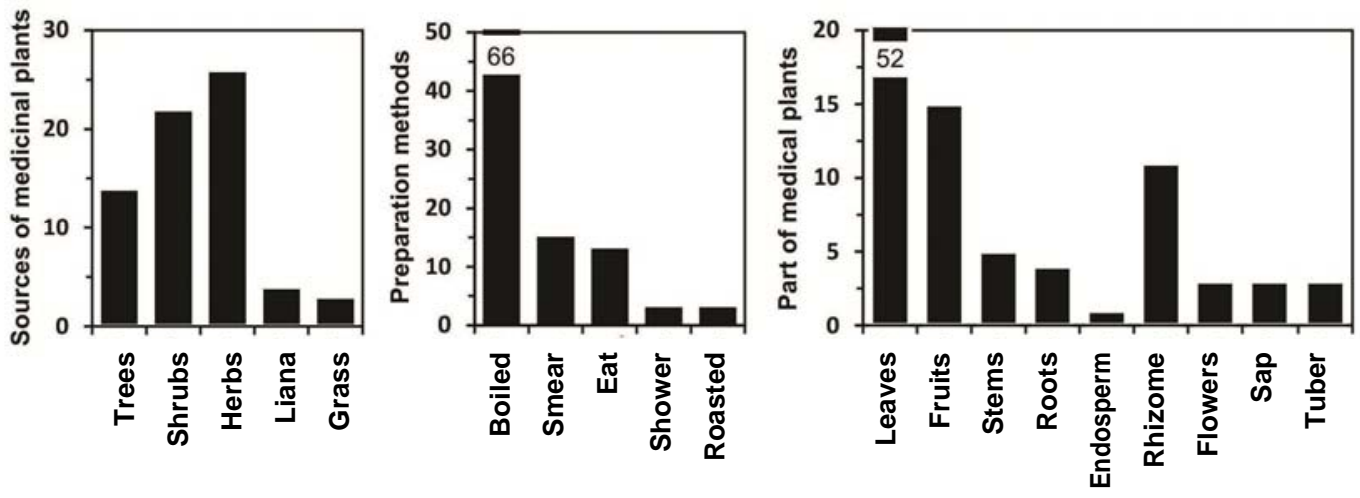

Figure 3. Medicinal plants in Turgo Hamlet based on: A. growth form, B. Processing, and C. parts of plants used

Species from Zingiberaceae, such as Dringo (Acorus calamus L.) was used to maintain body's immunity, tumeric kunir or kunyit (Curcuma domestica Val.) to cure liver disease, rheumatic, thypoid and diarrhea. Laos or lengkuas (Alpinia galanga (L.) Sw) to cure skin disease, temu giring (Curcuma heynena Vahl.) to lighten the skin for bride, Temuireng (Curcuma aeruginosa Roxb.) to increase appetite and to be used as vermicide. In addition, there were other species used for a variety of purposes, such as purple ginger or bengle (Zingiber purpureum Roxb.), jahe or ginger (Zingiber officinale Rosc.), and temulawak (Curcuma xanthorrhiza Roxb) (Table 1).

Family Asteraceae (8.7\%) was the second most used for medicines for various diseases (Table 2), consisting of bawukan (Ageratum conyzoides L.), marsh fleabane or beluntas (Pluchea indica L.), dewa (Gynura pseudochina L.), ireng-ireng (Eupatorium riparium Reg.), legetan (Synedrella nodiflora L.), and tempuyung (Sonchus arvensis L. ) (Table 1). The plants of this family were commonly found because they have made adaptation to tropical environment, and they have wide distribution and healing effect, so they are needed as solution to deal with the high price of modern medicines and the negative impacts of chemicals in modern medicines (Tjitrosoepomo 2010). Euphorbiaceae ranked third as medicinal plants (7.2\%), consisting of katuk (Sauropus androgynus L.), patikan cina (Euphorbia prostrata Aiton), patikan kerbau
(Euphorbia hirta L.), cassava or singkong (Manihot utilissima Crantz), and yodium (Jatropha multifida L.) (Tables 1 and 2). The plants of this family are used as medicines because they are commonly found in cultivation and in the wild.

Leaf is the most used part of plant for medicines, taken from 37 species (51.39\%) of plants (Figure 3), because it is the easiest part to get, easy too process, having healing effect, and its removal is not destructive to plants (Setyowati 2010). Leaf has high moisture content (70$80 \%)$, a place of photosynthesis, containing organic elements having medicinal effects and anti oxidants. Most green plants, such as avocado or alpukat (Persea americana Mill.), Indian pluchea or beluntas (Pluchea indica L.), mignonette vine or binahong (Anredera cordifolia (Ten.) Steenis), common comfrey or komprei (Symphytum officinale L.), dadap serep (Erythrina lithosperma (Hassk.) Merr), and daun dewa (Gynura pseudochina L) have leaves rich in carbohydrate, fiber, vitamin, and mineral (Table 1). Leaf was also the most used part of plant in traditional community of Gunung Simpang Nature Reserve, West Java, which was $31 \%$ of 74 species of medicinal plants, and also in the communities of Bulgaria and Gemeda, North Ethiopia, which was $50 \%$ of all total plants (Mesfin et al. 2013; Nedelcheva et al. 2013; Handayani 2015). 
Table 1. Medicinal plants in Turgo Hamlet: local name, scientific name, family, processing and health benefit/ health problems solved by each species

\begin{tabular}{|c|c|c|c|c|c|}
\hline Family & Scientific name & Local name & $\begin{array}{l}\text { Parts of } \\
\text { plant }\end{array}$ & Processing & $\begin{array}{l}\text { Health benefit or health } \\
\text { problems solved }\end{array}$ \\
\hline \multirow[t]{2}{*}{ Annonaceae } & Annona muricata L. & Sirsat & Leaf & Boiled & Uric acid, high blood pressure \\
\hline & Stelechocarpus burahol Blume & Kepel & Leaf & Boiled & Uric acid \\
\hline \multirow{3}{*}{ Apiaceae } & Centella asiatica (L.) Urban & Pegagan & Leaf & Boiled & Blood circulation \\
\hline & Eryngium foetidum L. & Musi Arab & Leaf & Smear & Vermicide \\
\hline & Foeniculum vulgare Mill. & Adas & Leaf & Boiled & Body’s immune system \\
\hline \multirow[t]{2}{*}{ Araliaceae } & Panax ginseng L. & Gingseng Jowo & Rhizome & Boiled & Body’s immune system \\
\hline & Acorus calamus L. & Dringo & Rod & Boiled & Body’s immune system \\
\hline \multirow[t]{5}{*}{ Arecaceae } & Cocos nucifera L. & Kelopo & Root & Boiled & Nerve system \\
\hline & & & Endosperm & $\begin{array}{l}\text { Directly } \\
\text { consumed }\end{array}$ & Itchy skin \\
\hline & Monstera pertusa (L.) de Vrise & Jalu Mampang & Leaf & Boiled & Appetite \\
\hline & Ageratum conyzoides L. & Wedusan & Leaf & Smeared & Skin cut \\
\hline & Eupatorium riparium (Regel) & Ireng-Ireng & Leaf & Boiled & Malaria \\
\hline \multirow[t]{4}{*}{ Asteraceae } & Gynura pseudochina Cass. & Dewa & Leaf & Boiled & Tumor \\
\hline & Pluchea indica (L.)Less. & Beluntas & Leaf & Boiled & Increasing breast milk \\
\hline & Sonchus arvensis L. & Tempuyung & Leaf & Boiled & Kidney stone \\
\hline & Synedrella nodiflora (L.)Gaerth & Legetan & Leaf & Boiled & Skin cut, toothache \\
\hline Araliaceae & Polyscias scutellaria Burm.F. & Mangko'an & Leaf & Boiled & Skin cut, diuretic \\
\hline Bassellaceae & Anredera cordifolia (Ten.) Steenis & Binahong & Leaf & Smeared & Skin cut \\
\hline Boraginaceae & Symphytum officinale L. & Comprei & Leaf & Mashed & Breathing problem, diabetes \\
\hline Bromeliaceae & Ananas comosus Mill. & Nanas & Fruit & Boiled & Fever \\
\hline Cactaceae & Epiphyllum sp. Haw & Sambung Otot & Leaf & Boiled & Nerve system \\
\hline Caricaceae & Carica papaya $\mathrm{L}$. & Kates & Leaf & Boiled & Diarrhea \\
\hline \multirow{2}{*}{ Cucurbitaceae } & Cucumis sativus L. & Ketimun & Fruit & Eat & High blood pressure \\
\hline & Sechium edule (Jacq) Sw. & Jipang & Leaf & Cook & High blod pressure \\
\hline \multirow{5}{*}{ Euphorbiaceae } & Jatropha multifida L. & Yodium & Leaf & Smear & Skin cut \\
\hline & Sauropus androgynus (L.)Merr. & Katuk & Leaf & Boiled & Increasing breast milk \\
\hline & Euphorbia prostate Aiton & Patikan Cina & All Species & Boiled & Diuretic, antipyretic \\
\hline & Euphorbia hirta L. & Patikan Kerbau & All Species & Boiled & $\begin{array}{l}\text { Asthma, diarrhea, kidney } \\
\text { infection }\end{array}$ \\
\hline & Manihot utilissima Crantz & Singkong & Leaf & Smear & Cold \\
\hline Equisetaceae & Equisetum debile Roxb & Sangkal Putung & Leaf & Boiled & $\begin{array}{l}\text { Cholesterol, skin cut, } \\
\text { Broken bones }\end{array}$ \\
\hline Fabaceae & Gliricidia sepium (Jacq.) Kunth ex Walp. & Kleresede & Leaf & Shower & Itchy Skin \\
\hline \multirow{2}{*}{ Liliaceae } & Aloe vera $\mathrm{L}$. & Lidah Buaya & Leaf & Smear & Skin burn \\
\hline & Allium sativum L. & Bawang Putih & Tuber & $\begin{array}{l}\text { Directly } \\
\text { consumed }\end{array}$ & Cholesterol \\
\hline Lamiaceae & Orthosiphon stamineus Benth. & Kumis Kucing & Leaf & Boiled & Kidney \\
\hline Lauraceae & Persea americana Mill & Alpokat & Leaf & Boiled & Uric acid, high blood pressure \\
\hline \multirow[t]{2}{*}{ Leguminosae } & Erythrina lithosperma (Hassk.) Merr & Dadap Serep & Leaf & Boiled & Fever \\
\hline & Leucaena leucocephala (Lam.)De Wit & Lamtoro gung & Leaf & Boiled & Skin cut, cancer, vermicide \\
\hline Loranthaceae & Loranthus sp. Jacq. & Kemladean & Parasit & Boiled & Cancer \\
\hline Malvaceae & Hibiscus similis $\mathrm{Bl}$. & Waru Gombong & Getah & Smear & Eye problem \\
\hline Marantaceae & Maranta arundinacea L. & Garut & Tuber & Roasted & Dyspepsia (gastritis) \\
\hline Melastomataceae & Medinella speciosa Reinw. Ex Blume & Parijoto & Flower & $\begin{array}{l}\text { Directly } \\
\text { consumed }\end{array}$ & Strengthening embryo \\
\hline Meliaceae & Swietenia macrophylla King. & Mahoni & Fruit & $\begin{array}{l}\text { Directly } \\
\text { consumed }\end{array}$ & Hemorrhoid \\
\hline Menispermaceae & Tinospora tuberculata (Thunb.) & Brotowali & Rod & Boiled & Appetite, diabetes \\
\hline Moraceae & Artocarpus heterophyllus Lam. & Nongko & Fruit & $\begin{array}{l}\text { Directly } \\
\text { consumed }\end{array}$ & Diarrhea \\
\hline \multirow[t]{2}{*}{ Musaceae } & Musa paradisiaca $\mathrm{L}$. & Pisang Kepok & Fruit & Roasted & $\begin{array}{l}\text { Kidney stone, gastritis, female } \\
\text { fertility }\end{array}$ \\
\hline & Musa textilis Nee & Pisang Raja & Sap & Smeared & Skin burn \\
\hline \multirow{3}{*}{ Myrtaceae } & Syzygium aromaticum L. & Cengkeh & Fruit & Boiled & Warming the body \\
\hline & Eugenia polyantha Wight. & Salam & Leaf & Boiled & Uric acid, cholesterol \\
\hline & Psidium guajava L. & Jambu klutuk & Leaf & Boiled & Diarrhea \\
\hline \multirow[b]{2}{*}{ Piperaceae } & Piper betle Linn & Suruh & Leaf & Boiled & Fluor albus, cough, body's odor \\
\hline & Piper betle var. nigra & Suruh ireng & Leaf & Boiled & Fluor albus, cough \\
\hline
\end{tabular}




\begin{tabular}{|c|c|c|c|c|c|}
\hline & Piper crocatum Ruitz \& Pav. & Suruh abang & Leaf & Boiled & High blood pressure \\
\hline & Saccharum officinarum L. & Tebu & Rod & $\begin{array}{l}\text { Directly } \\
\text { consumed }\end{array}$ & Kidney, eye inflammation \\
\hline \multirow[t]{4}{*}{ Poaceae } & Cynodon dactylon L. & Suket grinting & Leaf & Boiled & Uric acid \\
\hline & & & Root & Boiled & Nerve system \\
\hline & Imperata cylindrica L. & Alang alang & Leaf & Boiled & Skin cut \\
\hline & & & Rod & Boiled & Fever, uric acid \\
\hline Rubiaceae & $\begin{array}{l}\text { Morinda citrifolia L. } \\
\text { Paederia scandens L. }\end{array}$ & $\begin{array}{l}\text { Pace } \\
\text { Sembukan }\end{array}$ & $\begin{array}{l}\text { Leaf } \\
\text { Leaf }\end{array}$ & $\begin{array}{l}\text { Boiled } \\
\text { Cooked }\end{array}$ & $\begin{array}{l}\text { High blood pressure, uric acid } \\
\text { Increasing flatulence }\end{array}$ \\
\hline Rutaceae & Citrus aurantifolia (Christm.) Swingle & Jeruk pecel & Fruit & $\begin{array}{l}\text { Directly } \\
\text { consumed }\end{array}$ & Cough \\
\hline \multirow[t]{2}{*}{ Solanaceae } & Capsicum annum L. & Lombok & Fruit & Cooked & $\begin{array}{l}\text { Influenza, appetite, aphthous } \\
\text { stomatis }\end{array}$ \\
\hline & Solanum lycopersicum L. & Tomat & Fruit & $\begin{array}{l}\text { Directly } \\
\text { consumed }\end{array}$ & Reducing risk of Cancer \\
\hline Theaceae & Camellia sinensis L. & Teh & Leaf & Boiled & $\begin{array}{l}\text { Cancer, Asam Urat, } \\
\text { Rematik }\end{array}$ \\
\hline \multirow[t]{4}{*}{ Verbenaceae } & Clerodendrum japonicum L. & Pagoda & Flower & Boiled & Diuretic, antiseptic, Haemostatic \\
\hline & Vitex trifolia L. & Legundi & Leaf & Boiled & Uric acid \\
\hline & Zingiber purpureum Roxb. & Bengle & Rhizome & Boiled & Body’s immune system \\
\hline & Zingiber officinale Roscoe & Jahe & Rhizome & Boiled & cold, stiff muscles \\
\hline \multirow[t]{4}{*}{ Zingiberaceae } & Alpinia galangal (L.) Willd & Laos & Rhizome & Smeared & Skin fungus \\
\hline & Curcuma heyneana L. & Temugiring & Rhizome & Shower & Skin smoothing cream \\
\hline & Curcuma aeruginosa Roxb. & Temuireng & Rhizome & Boiled & Vermicide, appetite \\
\hline & Curcuma xanthorrhiza Roxb & Temulawak & Rhizome & Boiled & Liver, appetite \\
\hline
\end{tabular}

Fruit was also frequently used as medicines. The medicinal fruits were taken from 11 species (15\%) (Table 2), such as fennel or adas (Foeniculum vulgare Mill.), cabai or chili pepper (Capsicum annum L.), mahoni or magahony (Swietenia macrophylla King.), nangka or jackfruit (Artocarpus heterophyllus Lam.), and cheese fruit or pace or mengkudu (Morinda citrifolia L.). Fruits are used as medicines because they contain nutrient needed by human body such as potassium, pectin, beta-carotene and vitamin C. In addition, fruits also contain elements capable of cleansing food waste, has ready-to.-use energy (Gunawan 2007). Rhizomes of 8 species (11\%) were also used as medicines, such as, empon empon (Zingiberaceae): purple ginger of bangle (Zingiber purpureum Roxb.), tumeric or kunyit (Curcuma domestica Val.), Javanese ginger ot temulawak (Curcuma xanthorrhiza Roxb.), and jahe or ginger (Zingiber officinale Rosc.). Rhizomes are are used as medicines because they contain substances benefecial for health, such as zingeberene found in ginger (Z. officinale) which can be used to cure impotence and as beverage to warm the body (Figure 3).

Stem of four 4 species (5\%), namely brotowali (Tinospora crixspa L.), sweet flag or dringo (Acorus calamus L.), blady grass or rumput ilalang (Imperata cylindrica L.), and sugar cane or tebu (Saccharum officinarum L.) were also used as medicines. Roots of 3 species (4\%) namely Bermuda grass or rumput grinting (Cynodon dactylon), common comfrey or comprei (Symphytum officinale) and coconut or kelapa (Cocos nucifera L.) were used as medicines. Other organs, namely flower, sap, tuber, and the whole plant were rarely used. Medicinal flowers were taken from showy Asian grape or parijoto (Medinilla speciosa Reinw.) and bleeding heart or pagoda (Clerodendrum japonicum L.), while medicinal sap was taken from "king" banana or pisang raja (Musa paradisiaca L) and waru gombong (Hibiscus similis L). Medicinal tubers were taken from garlic or bawang putih (Allium sativum) and garut (Maranta arundinacea), while the the medicines from whole plants were found in prostate sandmat or patikan cina (Euphorbia prostate Aiton.) and asthma plant or patikan kerbau (Euphorbia hirta L). Only few people used parasitic plants and endosperm as medicines. Only 2 species (1\%) of parisitic plants, namely parasitic plant in tea or benalu teh (Loranthus sp.), and coconut or kelapa (Cocos nucifera) were sed as medicines (Table 1, Figure 3).

The people of Turgo Hamlet have used various processes of medicinal plant materials. Boiling (62\%), in order to dissolve the active substance into the water, was conducted for leaves of 28 species, rhizomes 5 of species, fruits of 4 species, stems of 3 species, roots of 1 species, and 1 species of parasitic plant. Smearing on skin (15\%) was done for leaves of 7 species, roots of 2 species, rhizomes of 1 species, and sap of 1 species, namely "king" banana pisang raja (Musa paradisiaca). Some parts of medicinal plants were consumed directly without processing (12\%), such as showy Asian grape or parijoto (Medinilla speciosa), coconut's endosperm (Cocos nucifera), stem of sugar cane (Saccharum officinarum), tuber of garlic (Allium sativum). Cooking of plant materials were rarely done (4\%). It was done for chilli pepper (Capsicum sp.), chayote or jipang (Sechium edule), and stinkvine or sembukan (Paederia scandens). Burning of plant materials was also rarely done (3\%). It was done for tuber of arrowroot or garut (Maranta arundinacea) and banana or pisang kepok (Musa paradisiaca) (Table 1). In addition, some plant materials were used for bathing (3\%), such as quickstick or klereside (Gliricidia sepium) and temugiring (Curcuma heyneana). 
Table 2. Percentage of families used as medicinal plants in Turgo Hamlet

\begin{tabular}{llll}
\hline Family & $\begin{array}{l}\text { Number } \\
\text { of genera }\end{array}$ & $\begin{array}{l}\text { Number } \\
\text { of species }\end{array}$ & \multicolumn{2}{l}{$\begin{array}{l}\text { Proportion } \\
\text { of } \\
\text { species }\end{array}$} \\
\hline Annonaceae & 2 & 2 & 2.90 \\
\hline Apiaceae & 3 & 3 & 4.35 \\
Araliceae & 1 & 1 & 1.45 \\
Arecaceae & 3 & 3 & 4.35 \\
Asteraceae & 6 & 6 & 8.70 \\
Bassellaceae & 1 & 1 & 1.45 \\
Boraginaceae & 1 & 1 & 1.45 \\
Bromeliaceae & 1 & 1 & 1.45 \\
Cactaceae & 1 & 1 & 1.45 \\
Caricaceae & 1 & 1 & 1.45 \\
Cucurbitaceae & 2 & 2 & 2.90 \\
Equisetaceae & 1 & 1 & 1.45 \\
Euphorbiaceae & 4 & 5 & 7.25 \\
Fabaceae & 1 & 1 & 1.45 \\
Lamiaceae & 1 & 1 & 1.45 \\
Lauraceae & 1 & 1 & 1.45 \\
Leguminosae & 2 & 2 & 2.90 \\
Portulacaceae & 1 & 1 & 1.45 \\
Liliaceae & 2 & 2 & 2.90 \\
Loranthaceae & 1 & 1 & 1.45 \\
Malvaceae & 1 & 1 & 1.45 \\
Marantaceae & 1 & 1 & 1.45 \\
Melastomataceae & 1 & 1 & 1.45 \\
Meliaceae & 1 & 1 & 1.45 \\
Menispermaceae & 1 & 1 & 1.45 \\
Moraceae & 1 & 1 & 1.45 \\
Musaceae & 1 & 2 & 2.90 \\
Myrtaceae & 3 & 3 & 4.35 \\
Piperaceae & 1 & 3 & 4.35 \\
Poaceae & 3 & 3 & 4.35 \\
Rubiaceae & 2 & 2 & 2.90 \\
Rutaceae & 1 & 1 & 1.45 \\
Solanaceae & 2 & 2 & 2.90 \\
Verbenaceae & 2 & 2 & 2.90 \\
Zingiberaceae & 3 & 7 & 10.14 \\
Total & $\mathbf{6 1}$ & $\mathbf{6 9}$ & $\mathbf{1 0 0}$ \\
\hline & & &
\end{tabular}

Table 3. Types of diseases cured with medicinal plants

\begin{tabular}{|c|c|}
\hline Types of diseases & Name of diseases and health benefit \\
\hline Mild diseases & $\begin{array}{l}\text { Skin cut, skin burn, aphthous stomatis, } \\
\text { stomach gas, fever, stiff muscles, } \\
\text { rheumatic, toothache, gastritis, hemorrhoid, } \\
\text { diuretic, fluor albus, influenza, skin } \\
\text { infection, diarrhea, cough, itchy skin, worm, } \\
\text { skin ulcer, skin fungus, eye inflammation, } \\
\text { warming the body, binding skin cut, } \\
\text { maintaining body's immune system, } \\
\text { maintaining brain health, increasing } \\
\text { fertility, anti inflammation, reducing body } \\
\text { odor and mouth odor }\end{array}$ \\
\hline Serious diseases & $\begin{array}{l}\text { Cholesterol, cancer, broken bones, diabetes, } \\
\text { kidney, diuretic, liver, uric acid, asthma, } \\
\text { kidney stone, nerve, high blood pressure, } \\
\text { diuretic, neutralizing intestines }\end{array}$ \\
\hline
\end{tabular}

The people of Turgo Hamlet had the capability of classifying diseases into two categories: mild and serious.
A disease is considered mild if it occurs to many people, and a disease is considered serious if it takes long time to heal it and it may cause death (Table 3). An example of mild disease is cold due to cold weather. The people cured this disease by consuming ginger ( $Z$. officinale) which contains curcumin, capable of proliferating $\mathrm{T}$ cells, so it has good prospect to increase immunity system (Varalakshmi et al. 2008). A serious disease, diabetes was generally cured with brotowali (Tinospora crispa) which contains alkaloid and flavonoid, capable of reducing sugar concentration in the blood.

In addition, the community of Turgo Hamlet had knowledge of the use of medicine for external application, namely smearing, and internal application, namely consuming (Table 3) An example of external application was the smearing of coral bush or daun yodium (Jatropha multifida) on the skin cut, because the leaves contain alkaloid compound beneficial for blood coagulation and thus useful for new cut. The leaves of common guava (Psidium guajava) was used to cure diarrhea, because the leaves contain astringent (a substance which can line mollusc intestine wall with a layer, protecting the wall from the stimulation of the intestine content), which is alkaline in nature, and capable of killing bacteria, Escherichia coli and Staphylococcus aureus.

The belief of Turgo Hamlet people on traditional healing is inherited from generation to generation, developed and governed together by the community. In Turgo Hamlet medicinal plants were usually found in homegardens, plantations, shrubs and in cultivated land. This fact is in line with the results of research by Hariyadi (2011) that medicinal plants are not taken from natural forest, but from human-dominated ecosystems, especially shrubs and cultivated land. The local wisdom of Turgo Hamlet community has long influenced the paradigm of the people on health, and indirectly has encouraged them to conserve biodiversity of tropical forest, which consist of various ecosystem types and serve as storage of biodiversity (Hidayat et al. 2010). More than 2,039 species of medicinal plants are found in tropical forest, useful to maintain health and to cure various diseases of human and cattle (Zuhud 2009). Therefore, the belief and knowledge of community of the use of medicinal plants must be developed and protected as heritage of traditional healing to maintain family health.

The people of Turgo Hamlet have used 69 plants species, from 36 families, growing in home gardens and in forest nearby, as medicinal plants. Their knowledge of medicinal plants has been inherited from generation to generation. The most used part of plant was leaf, followed by fruit, rhizome, stem, root, sap, flower, all parts or the whole plant, tuber, and endosperm. The medicinal plants consisted of several growth forms, namely tree, shrub, herb, liana and grass. The processes of medicinal plants were: boiled, directly smeared on skin, directly consumed, cooked, used for bathing, and burned.

It is obvious that the people of Turgo Hamlet have achieved health sovereignty for themselves and their families, based on their knowledge inherited from their ancestors, supported by the potential of natural resources in 
the village enabling them to do self healing for themselves and their families. In addition, they also have conducted ecosystem conservation through plant utilization for medicines. The community's knowledge will be improved if it is complemented with scientific research on the active substances of the medicinal plants, conducted by universities. So, sustainable research is needed in order to develop the knowledge qualitatively and quantitatively. It is also important to protect this local wisdom, so it will stay as the property of Indonesian nation.

\section{ACKNOWLEDGEMENTS}

Our thanks and great appreciation go to Turgo Hamlet community who have helped us by giving information of medicinal plants. Specifically, we thank Sudimejo, representing traditional midwife, Mujiwiyono, representing senior villagers, Muksimin, representing farmer group. We also appreciate our colleages, Hadi Sasongko, Purno Sudibya and Ardyan Pramudya Kurniawan, for their help in plant identification and completion of this study.

\section{REFERENCES}

Backer C.A. 1973. Atlas of 220 Weeds of Sugar-cane fields in Java. Indonesian Sugar Experiment Station, Pasuruan.

Bodeker G,. 2000. Indigenous Medical Knowledge: The Law and Politics of Protection: Oxford Intellectual Property Research Centre Seminar in St. Peter's College, 25 ${ }^{\text {th }}$ January 2000, Oxford

Gunawan A. 2007. Food Combining: Harmonious combination to be slim and healthy.: PT Gramedia PustakaUtama, Jakarta. [Indonesian]

Handayani, A. 2015. Utilization of medicinal plants by people around Gunung Simpang Nature Reserve, West Java; Proceeding of Nasional Seminary and Internasional Conference of Masyarakat Biodiversitas Indonesia, Bandung. 1 (6): 1425-143. [Indonesian]
Hariyadi B. 2011. The king medicines, the bargained medicine: medicinal plants and medicinal healing of Serampas tribe, Jambi, Biospecies 4 (2): 29-34.

Hidayat S., A Hikmat, E.A.M. Zuhud. 2010. Forest as Resources of Foods (Paper unpublish).

Idolo M., Ricardo M., Stefano M. 2010. Ethnobotanical and Phytomedicinal Knowledge in a long History Protected area, The Abrozzo, Lazzio and Molise national Park (Italian Apennines). J Ethnopharmacol 127 (2):379-395

Kandari LS, Ashish KG, Tripti N, Phondani PC. 2012. Ethnobotanical Knowledge of Medicine plant Among Tribal Communities in Orissa India. J. Forest Res 1 (1): 1-5.

Khan SM, Sue EP, Habib A, Daird MH. 2013. Sustainable and Conservation of Plant biodiversity in Montane Ecosystem: The Western Himalaya as a case study. J Ann Bot 112: 479-501.

Martin GJ. 2004. Ethnobotany: A 'People and Plant' Conservation Manual. Chapman and Hall, London.

Matthew WL, Heather Y, Christina K, Paul E, Aserat O, Rainer WB, Amber W. 2013. Local Knowledge of Plants and their uses among Women in the Bale Mountains, Ethiopia. Ethnobot Res Appl 11: 315339.

Mesfin K., Gebra T., Teklemichael T. 2013. Ethnobotanical study of traditional medicinal plant used by indigenous people of Gemad District Northen Ethiopia. J Med Plant Stud 1 (4): 32-37.

Muhammad M.M. 2000. Medical Miracles Prophet. Qultum Media, Jakarta.

Nedelcheva A. 2013. An ethnobotanical study of wild edible plants in Bulgaria. Eurasia J Biosci 7: 77- 94.

Pieroni A., Anely N., Avni H., B. Mustafa, Bruno S., Kevin C., Cassandra L.Q. 2014. Local knowledge on plant and domestic remedies in the mountain village of Peshkopia (Eastern Albania). J Mt Sci 11 (1): 180-194.

Setyowati, F. M. 2010. Ethnopharmacology and the use of plants among Dayak TunjungTribe in East Kalimantan. Media Litbang Kesehatan 3: 104-112.

Steenis CGGJ van. 1972. Mountain Flora of Java. E.J. Brill, Leiden.

Tjitrosoepomo G. 2010. Plant Morphology. GMU Press, Yogyakarta. [Indonesian]

Varalakshmi Ch, Mubarak Ali A, Pardhasaradhi BVV. 2008. Immunomodulatory effect of curcumin : in vivo. Intl J Immunol 8: 688-700.

Zuhud, EAM. 2009. The potential of tropical forest as the buffer for natural medicine material for the nation's health. Jurnal Bahan Alam Indonesia 6 (6) 227-232. [Indonesian] 